\title{
Prioritized Repeated Eliminations Multiple Access: A Novel Protocol for Wireless Networks
}

\author{
Greger Wikstrand \\ YAHM Sweden AB \\ Skifferv. 10 \\ SE-224 78 Lund \\ greger@cs.umu.se
}

\author{
Thomas Nilsson \\ Computing Science \\ Umeå University \\ SE-901 87 Umeå \\ nilsson@cs.umu.se
}

\author{
Mark S. Dougherty \\ Computer Science \\ Högskolan Dalarna \\ SE-791 88 Falun \\ mdo@du.se
}

\begin{abstract}
A new and simple MAC protocol is proposed. Each node transmits a burst with length sampled from a geometric distribution with parameter $q$ followed by a carrier sense slot. A node repeats the previous operations until it senses a busy slot. A node will access the channel and transmit its payload when it has sensed $h$ idle slots. The parameter $q$ can be adjusted for each node to achieve desired levels of relative priority with little impact on channel utilization and without a need for explicit knowledge about other traffic. Comparison to existing tree-splitting, carrier sensing and bursting protocols through analysis and simulations shows that our algorithm scales very well to the number of nodes. It has very high success probability, channel utilization and fairness. Extensions are provided for hidden terminal scenarios and are shown in simulations to provide adequate performance.
\end{abstract}

Index Terms-Access protocols, Wireless LAN, IEEE standards, ETSI standards, Computer network performance.

\section{INTRODUCTION}

Medium access control (MAC) protocols for decentralized wireless networks need to fulfill several conflicting goals at once: high channel utilization, high transmission success probability and high fairness. The protocol should also be simple to understand and implement. It should provide absolute and relative service differentiation. No centralized control should be required. High transmission success probability is especially important for broadcast transmissions where ARQ is difficult and ambiguous [1].

Existing MAC protocols use three basic mechanisms to allocate channel access: carrier-sensing, bursting and treesplitting. In this article we will make comparisons between a proposed protocol and an existing MAC protocol based on each of the three mechanisms. Nodes in carrier-sensing based protocols, such as the CSMA/CA based IEEE 802.11 standard, operate by repeatedly sensing the channel and then transmitting if enough slots are idle. Nodes in bursting-based protocols, such as EY-NPMA used in Hiperlan/1, actively try to win the channel by bursting, i.e. jamming the channel, longer than other nodes.Nodes in tree-splitting protocols, such as DQRAP-DW which is considered because it has similar performance characteristics, operate by means of a distributed depth-first tree-traversal.

We propose a novel bursting protocol for medium access control and compare it to protocols from each of the three groups as described below. The scope of the article is limited to the MAC layer of a single-hop decentralized wireless network.

\section{A. Carrier Sensing - IEEE 802.11}

The MAC algorithm used in IEEE 802.11 [2] is based on the Carrier Sense Multiple Access with Collision Avoidance (CSMA/CA) protocol. In CSMA/CA, nodes first sense the channel for a short time period called an Inter Frame Space (IFS). If the channel is busy or after a collision, nodes will sample a number of backoff slots from $U\{0, \mathrm{CW}\}$, where $\mathrm{CW}_{\text {min }} \leq \mathrm{CW} \leq \mathrm{CW}_{\max }$ is the contention window. The backoff counter is decremented, by one for each time slot, when the medium is sensed idle. Nodes will access the channel when their backoff is zero. The receiving node waits a short IFS (SIFS) and then transmits an ACK. After an unsuccessful transmission attempt (no ACK), the CW is doubled. After a successful attempt $\mathrm{CW}$ is set to $\mathrm{CW}_{\text {min }}$.

Optionally, an exchange of control frames prior to the data transmission, may be performed as follows. The sender starts by sending a Request To Send (RTS) and the receiver responds with a Clear To Send (CTS) packet, after first waiting a SIFS. Nodes in range of the receiver, the sender or both, will defer access during the completion of the frame exchange sequence.

In an IEEE 802.11 network, collisions allow involved nodes to adjust their contention windows. Information about conditions is gathered at high cost (collisions) but is not shared with non-colliding nodes. This leads to problems with fairness and low channel utilization.

The exponential doubling scheme favors the node which last succeeded and vice versa creating a fairness problem. A slower decrease of the contention window for successful nodes promote fairness and throughput. [3]

The channel utilization problem is caused by a high collision probability when non-optimal contention windows are used. A key problem in optimizing the size of the contention windows is getting an accurate estimate of the number of nodes. The collision probability depends only on the minimum contention window and the number of nodes; halving the minimum contention window is like doubling the number of users [4]. Both problems have been addressed through various contention window adaptation schemes, e.g. [5], [6]. Another approach is to minimize the impact of collisions on channel 
utilization by detecting and aborting colliding transmissions [1].

\section{B. Bursting - EY-NPMA}

Elimination yield non-pre-emptive priority prioritized multiple access (EY-NPMA), the channel allocation and conflict resolution protocol specified in Hiperlan/1 operates as follows [7]: After a successful transmission, nodes wait an IFS before the next contention starts. The contention operates in three phases: prioritization, elimination and yield. In the prioritization phase, nodes must sense the channel idle for a number slots equal to their priority $(0-4$, where 0 is the highest priority) before transmitting a priority assertion burst. Nodes with lower priority sense the channel busy and leave the contention while remaining nodes burst for a random number of slots, $X$, according to a geometric distribution, i.e. $X \sim \operatorname{Geom}\left(P_{E}\right)$ and

$$
P(X=k)= \begin{cases}p_{E}^{k}\left(1-p_{E}\right), & k=0,1, \ldots, m_{E S}-1, \\ p_{E} m_{E S} & k=m_{E S},\end{cases}
$$

where $p_{E}$ is the probability to burst in each elimination slot and $m_{E S}$ is the maximum burst time. After having verified their survival during a survival verification slot, nodes yield access for $U\left\{0, m_{Y S}\right\}$ slots. The node or nodes that sampled the lowest number will access the channel.

In the standard, slot-lengths are specified in terms of bit periods and are not the same for all types of slots. Here we use a uniform slot length $\tau$ for simplicity and comparability when analyzing the protocol. Default parameters in EY-NPMA, i.e. $p_{E}=0.5, m_{E S}=12$ and $m_{Y S}=9$, are non-optimal [8].

Blackburst is another bursting protocol, where burst lengths are assigned algorithmically based on sender priority [9].

\section{Tree Splitting - DQRAP-DW}

The basic tree-splitting algorithm works as follows [10]: when a collision occurs in a slot $i$ all nodes involved split into a number of subsets (e.g. by a random number or pre-assigned IDs), and the first subset retransmits in slot $i+1$ and the second in $i+2$ and so on. If another collision takes place between nodes in a subset then the subset recursively splits in two. The order in which the subsets are allowed to transmit can either be a breadth- or depth-first traversal. Fairness is guaranteed, not considering packet loss and interference, since each node transmits successfully exactly once in each round.

The distributed queuing random access protocol (DQRAP) was originally develop for wireline networks [11] but has been adapted for wireless networks with a central controller [12]. The operation of the protocol is quite complex, see [11] for a more detailed description. For purposes of comparison in the context of decentralized wireless networks we have developed a Distributed Wireless version called DQRAP-DW. It operates as follows: Under contention-free conditions nodes transmit a packet as soon as they have one available. If there is a collision and the packet is lost, i.e. no ACK, the nodes switch to contention based operation. After each fixed length dataslot, nodes involved in the collision randomly select one of two mini-slots to transmit a minimal packet. All non-transmitting nodes provide feedback in a micro-slot immediately following each mini-slot. If a collision is detected they transmit a NACK and the involved nodes place themselves in the resolution queue (RQ). If there was a single node, i.e. no NACK, it is placed in the transmission queue (TQ). After the two minislots the first node in the TQ access the channel. Nodes maintain the lengths of both queues and their own positions in them.

CARMA-FS is an another tree-splitting protocol. In CARMA-FS the controller is designated dynamically by the winning sender in a depth first traversal of the tree which is truncated at first-success [13].

\section{Outline of this Article}

In the following sections, we propose a novel MAC algorithm and evaluate it analytically. Simulation results are presented in Section IV. Then in Section V a priority mechanism for relative service differentiation is described and evaluated analytically. Two alternative mechanisms for coping with hidden terminals are presented and evaluated through simulations in Section VI. Finally the results are discussed and the article is concluded.

\section{Proposed Algorithm - PREMA}

None of the described protocols fulfill all of the requirements that we posed on a MAC protocol in the introduction. We propose a new and simple bursting protocol, named Prioritized Repeated Eliminations Multiple Access (PREMA).

The channel is assumed to be slotted and each sender is capable to perform one of the two following actions in each slot: a carrier sense operation or perform a short burst or jamming transmission. Senders randomly select which action $A$ to perform in each slot $i$ with $P\left(A_{i}=t x\right)=q_{i}$ and $P\left(A_{i}=c s\right)=1-q_{i}=p_{i}$. If the sender senses a busy channel it has lost and defers access. If it senses an idle channel it increases a counter $i d l e S l o t s$. When $i d l e S l o t s=h$ it is considered to have won and starts transmitting. PREMA is described in the form of pseudo-code in Algorithm 1.

An example of the algorithm in operation is given in Fig. 1. In the example $n=6, h=4$ and $q=0.5$. Initially all nodes wait a $T_{i f s}$ before randomly performing a carrier sense $\left(A_{i}=\mathrm{cs}\right)$ or an elimination burst transmission $\left(A_{i}=\mathrm{tx}\right)$. In the example $\mathrm{S}_{2}$ and $\mathrm{S}_{5}$ sense a busy channel and leave the contention. In the following slots $S_{3}$ and $S_{6}$ are eliminated. The remaining nodes $S_{1}$ and $S_{4}$ both survive the first elimination and enter the second where $S_{1}$ is eliminated. $\mathrm{S}_{4}$ performs two additional eliminations until idleSlots $=h$ and access is allowed.

\section{Analytical Performance Evaluation}

In the following, the proposed algorithm is evaluated and optimal parameters are derived. The following assumptions are made: The channel operates under saturation conditions. There are no hidden terminals. Channel noise etc. does not affect the results. All nodes have the same priority. All packets have the same length. 

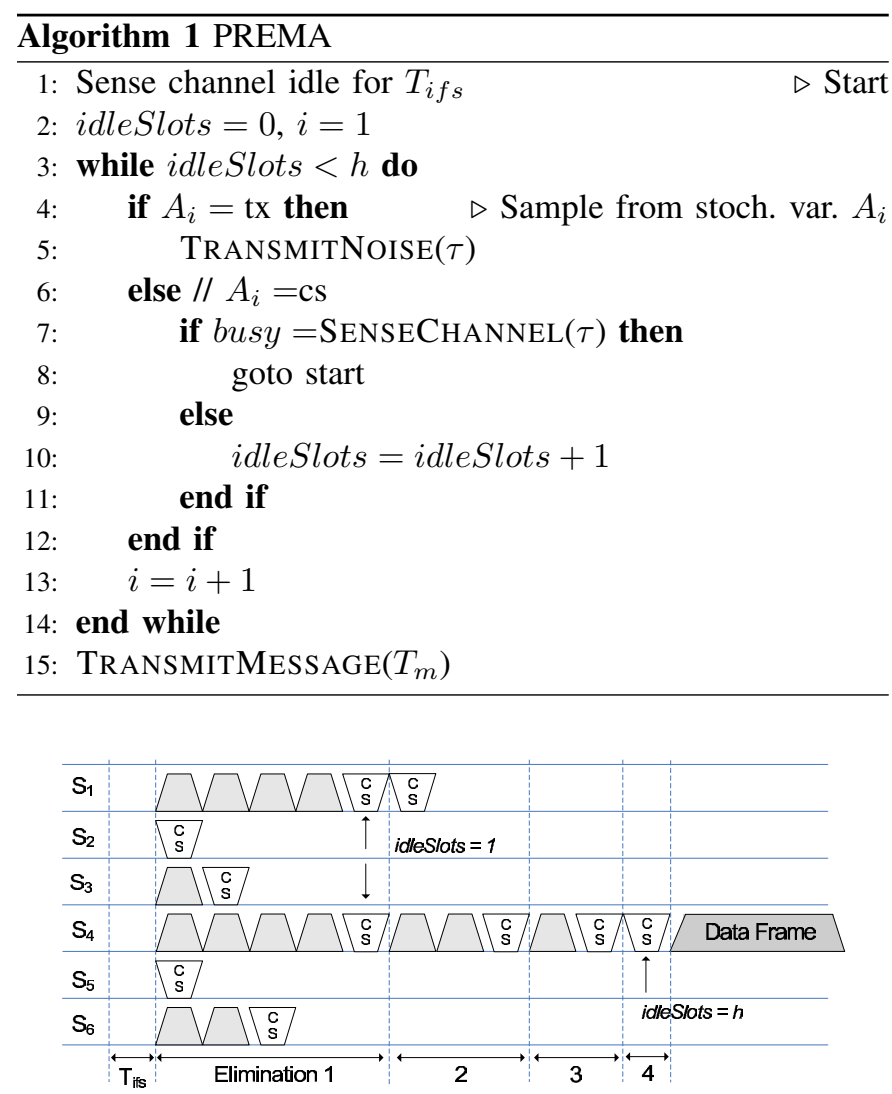

Fig. 1. PREMA scenario with six nodes. Grey trapezoids are elimination bursts, white are carrier sense operations and the wide dark grey trapezoid is the main transmission.

TABLE I

Notation AND VALUES USED IN THE ARTICLE. TIMING VARIABLES ARE GIVEN IN $\mu$ S.

\begin{tabular}{ll}
\hline \hline Parameter & Description \\
\hline$q=0.5$ & $P(A=t x)$ \\
$p=1-q$ & $P(A=c s)$ \\
$n$ & Number of nodes \\
$h=4$ & PREMA threshold \\
$\tau=20$ & Slot time \\
$T_{\text {other }}$ & $T_{i f s}+T_{a c k}+T_{\text {sifs }}+T_{p h y}+T_{m a c}$ \\
$T_{m a c}$ & Mac frame overhead \\
$T_{p h y}=96$ & Synchronization time for physical header \\
$T_{m}=6050$ & Tx time for data payload \\
$T_{a c k}=56$ & Tx time for ACK frame \\
$T_{c t s}=56$ & Tx time for CTS frame \\
$T_{r t s}=80$ & Tx time for RTS frame \\
$T_{i f s}=(h+1) \tau$ & Required idle time before new contention phases \\
$R=2 \cdot 10^{6}$ bps & Physical data rate \\
$R_{t x}$ & Transmission range \\
$R_{c s}$ & Carrier sense range \\
$p_{i}$ & Transmission probability of nodes in class $i$ \\
$\hat{r}_{i 1}$ & Desired relative priority of nodes in class $i$ \\
$n_{i}$ & Number of nodes in class $i$ \\
\hline
\end{tabular}

\section{A. Single Elimination}

The reasoning in this section parallels that of our analysis of the elimination phase in EY-NPMA [8]. In each elimination, the nodes that burst for the longest time will survive. The expected length of the longest burst among $n$ nodes is [14]

$$
\mu_{n, 1}=-\sum_{k=1}^{n}\left(\begin{array}{l}
n \\
k
\end{array}\right) \frac{(-1)^{k}}{1-q^{k}} .
$$

The exact form (1) is unwieldy for large $n$. An approximation of the expected length of the elimination phase is

$$
\tilde{\mu}_{n, 1} \approx \log _{q^{-1}} n+\gamma / \log \left(q^{-1}\right)+0.5
$$

excluding the error term and where $\gamma=0.577 \ldots$ is the Euler constant [14]. In the numerical examples in this article, the approximation is used when $n>50$.

The probability that $m$ out of $n$ nodes survive the elimination phase is [15]

$$
p_{m, 1}(n)=p^{m}\left(\begin{array}{c}
n \\
m
\end{array}\right) \sum_{k=0}^{n-m}\left(\begin{array}{c}
n-m \\
k
\end{array}\right) \frac{(-1)^{k}}{1-q^{k+m}} .
$$

An approximation is useful for large $n$

$$
\tilde{p}_{m, 1}(n) \approx \frac{p^{m}}{\log \left(q^{-1}\right)}\left(\frac{1}{m}+\delta_{m}\left(\log _{q^{-1}}(n)\right)\right),
$$

excluding the error term and where $\delta_{m}(x)=1 / m$ ! . $\sum_{j \neq 0} \Gamma\left(m-2 j \pi i / \log \left(q^{-1}\right)\right) e^{2 j \pi i x}$ and $\Gamma$ is the gamma function [15]. In the numerical examples in this article, the exact form is used when $n<10$.

\section{B. Repeated Eliminations}

The probability that $m$ out of $n$ nodes survive the $k$ th elimination is easily obtained by recursively extending (3). We obtain,

$$
p_{m, k}(n)= \begin{cases}\sum_{i=m}^{n} p_{i, k-1}(n) p_{m, 1}(i) & k>0, \\ 1 & n=m, k=0, \\ 0 & \text { otherwise. }\end{cases}
$$

In particular, the transmission success probability is equal to the probability of there being only one node left after $h$ eliminations

$$
p_{S}=p_{1, h}(n)
$$

The expected length of the $k$ th elimination is similarly given by

$$
\mu_{n, k}=\sum_{i=1}^{n} p_{i, k-1}(n) \mu_{i, 1}
$$

1) Approximation: Substituting numerical values shows that for most values of $n, p_{S} \approx 0.721$ when $q=.5$ and $h=1$. A similar property was observed experimentally in EYNPMA [16]. The probability that a node is the single winner after detecting $h$ idle slots is

$$
p_{S} \approx 1-\left(1-p_{1,1}(n)\right)^{h} \stackrel{q=0.5}{\approx} 1-(1-0.721)^{h} .
$$

Numerical results from the approximation (8) are close to that of the the exact formulation (6), e.g. within \pm 0.02 for $n=50$. 


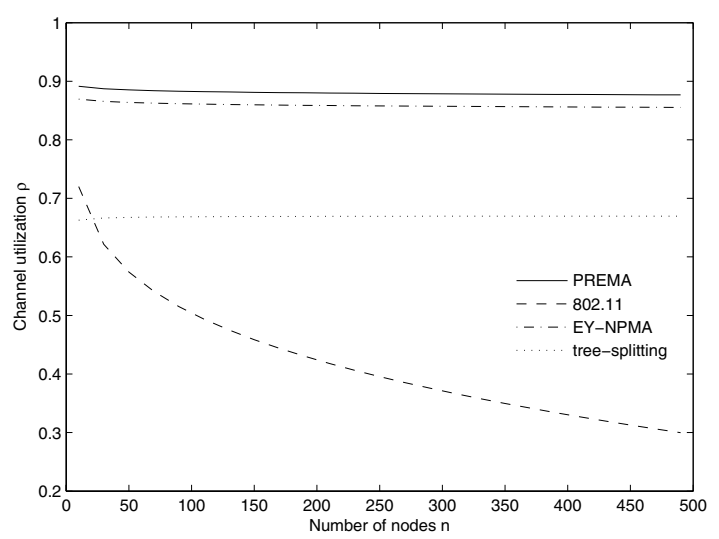

(a) Analytical

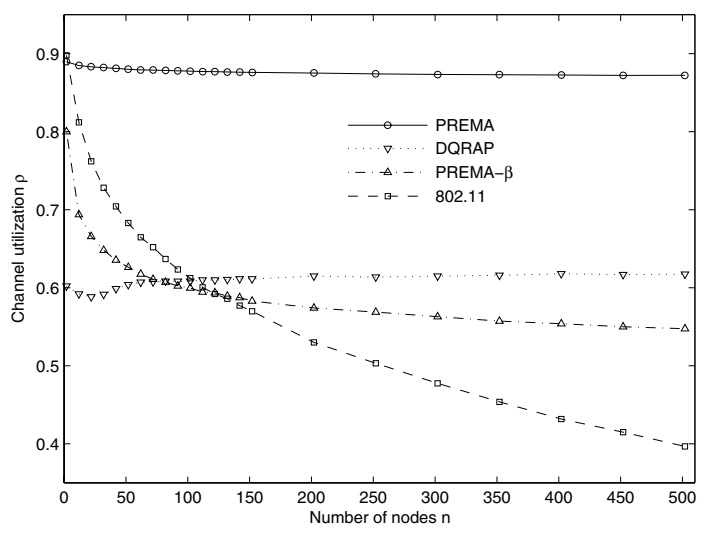

(b) Simulated

Fig. 2. Channel utilization of DQRAP-DW, IEEE 802.11, PREMA and a generic tree-splitting algorithm. $n=2, \cdots, 500$.

\section{Channel Utilization}

The average channel utilization $\rho$ is given by the duration of useful data transmission $T_{m}$ in a cycle divided by the duration of the the cycle multiplied by the probability of a successful transmission in the cycle, i.e.

$$
\rho=\frac{T_{m} p_{S}}{\tau \sum_{k=1}^{h} \mu_{n, k}+T_{m}+T_{\text {other }}} .
$$

The channel utilization is compared to that of a generic treesplitting protocol [17], EY-NPMA [18] and the IEEE 802.11 protocol [19] in Fig. 2(a). Timing parameters are presented in Table I. Our values of EY-NPMA are based on using the same slot length, inter frame space, packet lengths and overhead as for the other protocols.

\section{Optimal Parameters}

Using standard numerical techniques and (9), it is a simple matter to calculate optimal parameters $h$ and $q$, given certain other parameters as found in Table I. For a given $h$, there is a corresponding optimal $q$. Lower $h$ corresponds to higher $q$ and vice versa, see Fig. 3. For a wide range of $n, h=4$ and $q=0.5$ seem to be acceptably close to optimal, see Table II. In the following, we shall use $h=4$ and $q=0.5$ unless otherwise stated.

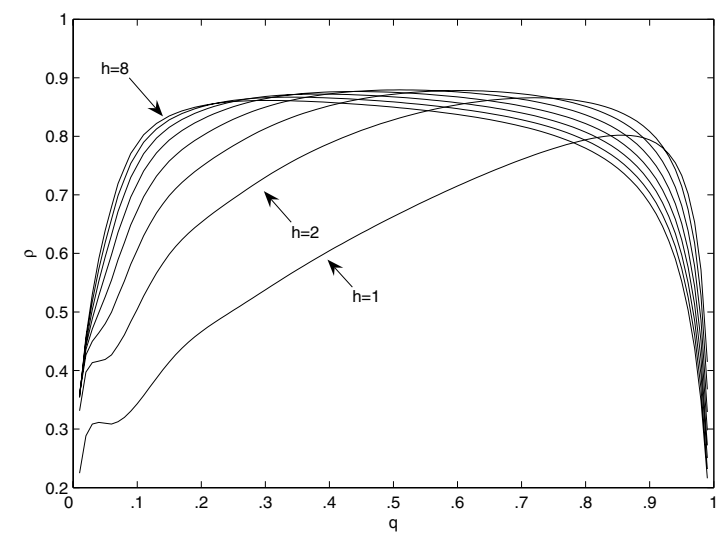

Fig. 3. $\rho$ as a function of $h$ and $q, n=40$.

TABLE II

PARAMETERS AND PERFORMANCE FOR PREMA AND EY-NPMA

\begin{tabular}{rrlrllr}
$n$ & $h, m_{E S}{ }^{1}$ & $q, p_{E}{ }^{2}$ & $m_{Y S^{3}}$ & $\rho$ & $p_{S}$ & $\mu^{4}$ \\
\hline 10 & 4 & 0.5 & & 0.87843 & 0.99041 & $15.063^{5}$ \\
& 4 & 0.525 & & 0.87871 & 0.99257 & $15.695^{6}$ \\
& 12 & 0.5 & 9 & 0.86953 & 0.96484 & $9.6556^{7}$ \\
& 9 & 0.60823 & 13 & 0.87574 & 0.98179 & $13.133^{8}$ \\
\hline 70 & 4 & 0.5 & & 0.87153 & 0.99052 & $17.801^{5}$ \\
& 4 & 0.5015 & & 0.87153 & 0.99066 & $17.849^{6}$ \\
& 12 & 0.5 & 9 & 0.8625 & 0.96482 & $12.388^{7}$ \\
& 10 & 0.50003 & 16 & 0.86738 & 0.97901 & $15.43^{8}$ \\
\hline 130 & 4 & 0.5 & & 0.86927 & 0.99052 & $18.694^{5}$ \\
& 5 & 0.42671 & & 0.86964 & 0.99249 & $19.233^{6}$ \\
& 12 & 0.5 & 9 & 0.86026 & 0.9648 & $13.262^{7}$ \\
& 10 & 0.47943 & 16 & 0.86521 & 0.97761 & $15.801^{8}$ \\
\hline
\end{tabular}

\footnotetext{
${ }^{1} h$ for rows with PREMA, $m_{E S}$ for rows with EY-NPMA

${ }^{2}$ Bursting probability

3 Only for rows with EY-NPMA

4 Total average length of contention in slots

5 PREMA, default parameters, ${ }^{6}$ PREMA, optimal parameters

7 EY-NPMA, default parameters, ${ }^{8}$ EY-NPMA, optimal parameters
}

We compare PREMA to EY-NPMA with optimal and default parameters in Table II and Table III. In Table II the performance of each protocol is shown in terms of $\rho, p_{S}$ and the average contention length $\mu$ for various $n$. We note that $\rho$ and $p_{S}$ are marginally higher for PREMA than for EYNPMA and higher for the optimal rather than the default parameters. The difference is small in $\rho$ and larger in $p_{S}$. In Table III we see that there is a price to pay for the higher $p_{S}$ of PREMA for small packets. The channel utilization is slightly lower for PREMA for small packets. The crossing point is around $m=3500$. In practice however the differences in $\rho$ are negligible. The differences in $p_{S}$ are more pronounced and will have a large impact on performance in broad- and multicast traffic, where detection of collided packets is difficult.

\section{Simulation Results}

In this section we first validate the analytical model with simulations under saturation conditions. We then extend the study by performing simulations under variable load and compare the performance with IEEE 802.11, PREMA- $\beta$ (described in Section VI) and DQRAP-DW in terms of channel utilization and fairness. 
TABLE III

PARAMETERS AND PERFORMANCE FOR PREMA AND EY-NPMA

\begin{tabular}{rrlrllr}
$T_{m}$ & $h, m_{E S}{ }^{1}$ & $q, p_{E}{ }^{2}$ & $m_{Y S}{ }^{3}$ & $\rho$ & $p_{S}$ & $\mu^{4}$ \\
\hline 20 & 4 & 0.5 & & 0.023673 & 0.99052 & $17.342^{5}$ \\
& 2 & 0.43236 & & 0.025659 & 0.88078 & $9.8261^{6}$ \\
& 12 & 0.5 & 9 & 0.026498 & 0.96483 & $11.912^{7}$ \\
& 4 & 0.25431 & 7 & 0.028499 & 0.90756 & $7.3451^{8}$ \\
\hline 2020 & 4 & 0.5 & & 0.70531 & 0.99052 & $17.342^{5}$ \\
& 3 & 0.51866 & & 0.70693 & 0.97502 & $14.804^{6}$ \\
& 12 & 0.5 & 9 & 0.71436 & 0.96483 & $11.912^{7}$ \\
& 7 & 0.41806 & 11 & 0.71596 & 0.96207 & $11.219^{8}$ \\
\hline 4020 & 4 & 0.5 & & 0.82325 & 0.99052 & $17.342^{5}$ \\
& 4 & 0.46184 & & 0.82366 & 0.98633 & $16.198^{6}$ \\
& 12 & 0.5 & 9 & 0.82031 & 0.96483 & $11.912^{7}$ \\
& 8 & 0.46909 & 14 & 0.82272 & 0.97376 & $13.399^{8}$ \\
\hline 6020 & 4 & 0.5 & & 0.87218 & 0.99052 & $17.342^{5}$ \\
& 4 & 0.51228 & & 0.8723 & 0.99162 & $17.673^{6}$ \\
& 12 & 0.5 & 9 & 0.86327 & 0.96483 & $11.912^{7}$ \\
& 10 & 0.52142 & 15 & 0.86817 & 0.97911 & $14.966^{8}$ \\
\hline
\end{tabular}

12345678 See Table II

\section{A. Simulation Model}

We have used the GloMoSim simulator environment [20]. For physical layer modeling we use a simple two-ray propagation model with no additional fading. Our main interest is to evaluate the characteristics of the algorithms from a capacity sharing point of view and therefore a simpler radio model is assumed. The simulation area is quadratic with sides 200 meters long. The number of senders $n$ is varied for the different simulation scenarios. No mobility is assumed and the position of each sender is randomly set within the simulation area. For more simulation parameters see Table I.

1) Rate: Each sender transmits packets according to a Poisson process with rate $\lambda$ packets per second. The receiver for each transmission is uniformly chosen from the neighbors of each sender. The model will provide a mix of traffic to close and far nodes controlling for the capture effect.

2) $A R Q$ : One of the motivations for using ARQ in IEEE 802.11 is to detect collisions and adjust the contention windows accordingly. In PREMA the probability for two or more senders to win the same contention phase, and start transmitting simultaneously, with a threshold of $h=4$ is very small, see Section III. The motivation for using ARQ in PREMA, for the same reason as in IEEE 802.11, does not hold. However, for comparison reasons we assume that the stop-and-wait ARQ is applied in all protocols.

3) Feedback: We make the following assumptions for the size and feedback capability of each time slot, the short time slot is used for $(0 / m)$ binary feedback, i.e. a sender may perform a carrier sense in this slot to detect a busy or idle channel. The long time slots, used in DQRAP-DW, provide an additional level of feedback and the sender may detect an idle slot, a slot carrying a correct signal or a collision, socalled ternary feedback $(0 / 1 / m)$. The size of this slot must be longer since a receiver should be able to decode parts of the signal to determine if the slot carries correct information.

4) Metrics: We use two metrics: channel utilization or throughput and Jain fairness index. The channel utilization is the same as the normalized aggregate throughput i.e. the aggregated throughput divided by the modulation rate. The Jain fairness index is a measure of fairness, i.e. the equalness of the division of resources. A Jain fairness index of one indicates absolute fairness. Lower values, minimum zero, indicate increasing unfairness. The Jain fairness index is defined as [21]

$$
F_{\text {Jain }}=\frac{\left[\sum_{i} x_{i}\right]^{2}}{n \sum_{i} x_{i}^{2}}
$$

where in our case $x_{i}$ is the throughput of node $i$.

\section{B. Results}

Fig. 2(b) shows the simulated channel utilization for PREMA, PREMA- $\beta$, DQRAP-DW and IEEE 802.11 under saturated conditions for increasing number of contending senders. PREMA- $\beta$ is an extension for hidden terminals and is described in Section VI. PREMA and DQRAP-DW show stable performance when increasing $n$ while the channel utilization drops for IEEE 802.11 and PREMA- $\beta$.

There is a small dip in the DQRAP-DW curve when $n$ is small. This is a result of the feedback mechanism. A node can only provide feedback for one mini-slot if it is transmitting in the other slot. If all nodes choose the same mini-slot, and there are no nodes in the resolution or transmission queues, then there will not be any node providing feedback for that particular slot. This is more likely when $n$ is small. The result is incorrect feedback leading to incoherent queue states and ultimately to data slot collisions.

Apart from that, the simulation results verify the analysis. Analytical results for PREMA and IEEE 802.11 correspond well to the corresponding simulation results. The analytical model overestimates the simulation results for PREMA slightly. The analytical model underestimates the channel utilization for IEEE 802.11 when there are many nodes. This is because the model does not consider all relevant parameters that effect the performance of IEEE 802.11, for example the Extended IFS (EIFS). The generic tree-splitting approximation used in Fig. 2(a) is a good but optimistic predictor for the simulated DQRAP-DW results.

Fig. 4 shows how fairness converges over time towards a steady-state under saturated conditions for DQRAP-DW, PREMA- $\beta$, PREMA and IEEE 802.11. DQRAP-DW almost immediately reaches perfect fairness. A result of the global coherent state with FIFO queues. The other three protocols converge towards roughly the same level of fairness but IEEE 802.11 converges more slowly. The problems with fairness in IEEE 802.11 were discussed in the introduction. It is interesting to note that although fairness converges more or less slowly for each protocol the relative ordering remains the same. The reader should also note that the number of packets served is not the same for all protocols, since the throughput is not the same. Using the number of packets served instead of elapsed time does not change the relative ordering of the protocols.

Fig. 5 shows the aggregated throughput when increasing the aggregated load for 100 nodes. The aggregated throughput of PREMA, PREMA- $\beta$ and DQRAP-DW increases linearly until 


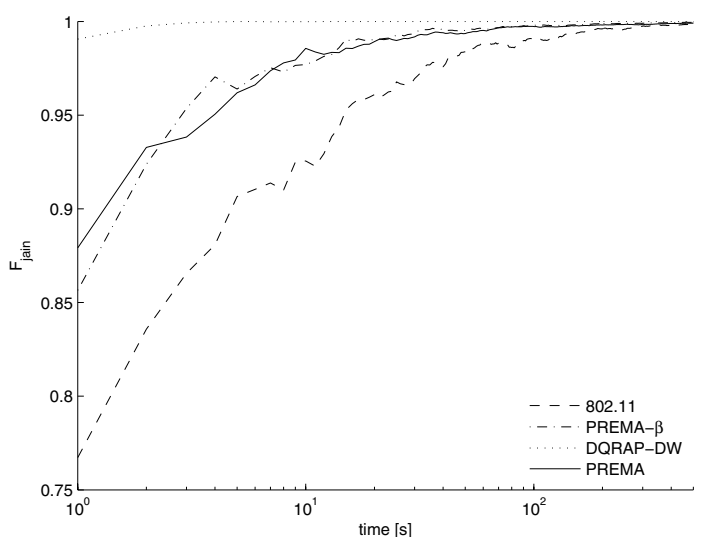

Fig. 4. The Jain fairness index as a function of time.

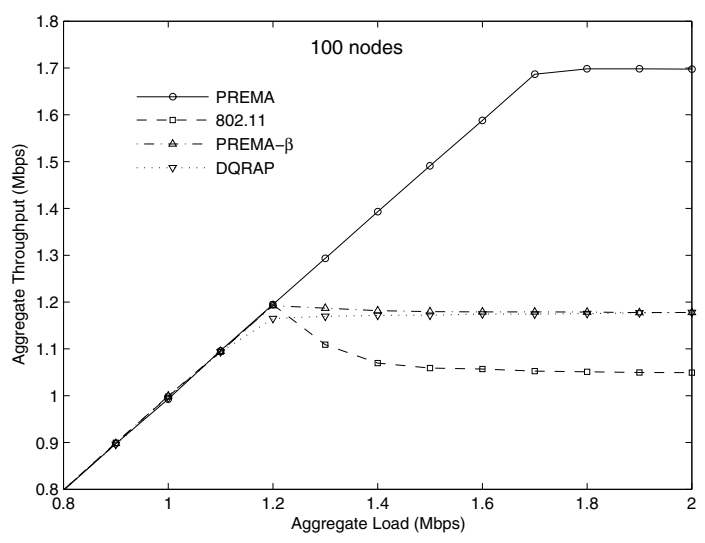

Fig. 5. Aggregate throughput as a function of traffic load for $n=50$ and $n=100$.

saturation is reached and then remains stationary. On the other hand, the throughput of IEEE 802.11 reduces asymptotically after the saturation point is reached. The saturation point is reached much later for PREMA.

\section{Priority Mechanism}

In this section, we first review a few existing solutions for service differentiation in CSMA/CA networks. We then extend PREMA with a simple priority mechanism by introducing priority vectors with the purpose of achieving service differentiation through relative prioritization. The performance is compared with optimal contention windows for IEEE 802.11 and fixed contention windows also for IEEE 802.11 (like IEEE 802.11e) through a simple analysis and simulations.

Three basic service differentiation mechanisms have been proposed for IEEE 802.11 networks: setting different CWs [22], [23], allowing high priority traffic to use a shorter IFS (called prioritization in EY-NPMA and AIFS in IEEE 802.11e), and limiting the maximum transmission time. All of these mechanisms have been combined in IEEE 802.11e in the form of service classes which each gets a certain combination of the aforementioned factors [24].

Implementing an AIFS-style prioritization mechanism in PREMA is trivial and we will not delve further on the topic here. Regardless of if such a mechanism is implemented or not it is desirable to implement relative prioritization to distinguish between traffic with the same absolute priorities.

In IEEE 802.11 such pre-emptive prioritization can be achieved via two mechanisms: setting optimal contention windows for each priority class or setting fixed contention windows for each class.

\section{A. Optimal Contention-Window}

Different CWs can be set like this [23]: First calculate the optimal $p$-persistent transmission probability for each priority class using (17) from [23]

$$
p_{i}=\frac{\hat{r}_{i 1} \cdot p_{1}}{\hat{r}_{i 1} \cdot p_{1}+1-p_{1}}
$$

where $\hat{r}_{i 1}$ is the desired relative priority of class $i$ compared to a class 1 or basic priority flow and $p_{1}$ is chosen to maximize the channel utilization according to (29), (30) and (39) from [23]

$$
\begin{aligned}
p_{1}=\frac{x}{1+x} \stackrel{\mathrm{x} \text { is small }}{\approx} x= \\
\frac{\sqrt{2 \tau} / \sqrt{\sum_{i=1}^{P} n_{i} \hat{r}_{i 1}{ }^{2}-\sum_{i=1}^{P} n_{i} \hat{r}_{i 1}^{2}}}{\sqrt{\left(T_{m}+T_{\text {other }}\right)}},
\end{aligned}
$$

where $P$ is the number of priority classes and $n_{i}$ is the number of nodes in class $i$. Finally we obtain the desired contention window sizes using (41) from [23]

$$
C W_{i}^{*}=\left\lfloor\frac{2}{p_{i}^{*}}-2.0\right\rfloor .
$$

\section{B. Fixed Contention-Window}

An alternative mechanism is to use fixed, pre-defined contention windows for each priority class as in IEEE 802.11e. Xiao suggests creating two priority classes by assigning different initial contention windows $C W_{\min , i}$, different windowincreasing factors $\sigma_{i}$ and different maximum back off stages $m_{i}$ so that $C W_{\max , i}=C W_{\min , i} \sigma_{i}^{m_{i}}$ for priority class $i$ [22]. We use two priority classes with Class 1 defined by $C W_{\min , 1}=4, \sigma_{1}=1.7$ and $m_{1}=4$ and Class 2 defined by $C W_{\min , 2}=8, \sigma_{2}=2$ and $m_{2}=7$ as suggested by Xiao [22].

\section{Proposal}

In PREMA, the probability of staying in the contention if a slot is busy is

$$
P(\text { stay })=P(\text { stay } \mid A=c s) p+P(\text { stay } \mid A=t x) q .
$$

In the basic mechanism $P($ stay $\mid A=c s$, channel $=$ busy $)=0$ and $P($ stay $\mid A=t x)=1$ so $P($ stay $\mid$ channel $=$ busy $)=q=$ 0.5 . Adjusting either of the two "staying" probabilities, e.g. by giving nodes "extra lives", would mean that there would be a discrepancy between the information available in the wireless medium and the internal state of some nodes.

That leaves a mechanism based on adjusting $q$ for each node. Since the number of slots in a contention is unknown 
beforehand having unequal but fixed $q$-values would make it hard to predict the final priority of a class of nodes. Instead we have chosen to work by assigning different $q$-values to each node and slot.

It is easily verified, e.g. through outcome-tree analysis, that a node with $\mathbf{q}=\{1,0.5,0.5, \ldots\}$ will have a doubled probability of winning the first elimination. It will behave as two virtual nodes. The base priority vector is $\mathbf{q}=\{0.5,0.5, \ldots\}$. The probability of winning, i.e. the relative priority, can be calculated for arbitrary $\mathbf{q}-$ vectors as

$$
r_{i 1}=0.5^{-k} \prod_{j=1}^{k} \mathbf{q}_{i}(j)
$$

where $k$ is the length of the elimination. Note that elements of $\mathbf{q}_{i}(j)$ where $j>k$ will not have any effect on the final outcome. For simplicity of analysis we assume that $\mathbf{q}_{i}(j)=$ 0.5 for any $j \lesssim k$.

The total channel utilization for a PREMA network with priorities is given by substituting the total number of virtual nodes $n^{\prime}=\sum_{i=1}^{n} r_{i 1}$ for $n$ in (9). Channel utilization for node $i$ is

$$
\rho_{i}=\frac{r_{i 1}}{n^{\prime}} \rho\left(n^{\prime}\right)
$$

\section{Results}

We have compared the three approaches analytically: variable $q$ in PREMA, and optimal and fixed CW in IEEE 802.11. We consider two classes of nodes with class 1 having base priority and class 2 having priority $r_{21}=5.5895$. The choice was prompted by the fact that this is the relative priority obtained between two nodes using Xiao's method. The channel utilization for PREMA was calculated using (15). The optimal CWs were calculated using Ge's method as outlined in (10)(12). The channel utilization for both fixed and optimal CW was calculated using Xiao's method [22].

We consider two scenarios, presented in Fig. 6. In both, the total channel utilization is robust for PREMA and optimal $\mathrm{CW}$ although slightly better for PREMA. The overall channel utilization drops rapidly for IEEE 802.11 with fixed CW.

In the first scenario, Fig. 6(a), the number of base priority nodes is fixed, $n_{1}=50$, while increasing number of high priority nodes are injected in the network. In PREMA and optimal CW an increasing share of the channel goes to the high priority class. The base priority class retains some share. With fixed $\mathrm{CW}$ almost all of the diminishing channel utilization goes to the high priority class.

In the second scenario, Fig. 6(b), both classes grow at equal rates. PREMA and optimal $\mathrm{CW}$ maintain a constant relation between the classes while constant $\mathrm{CW}$ leads to an ever decreasing share for the base priority class. PREMA is slightly better than optimal $\mathrm{CW}$ at maintaining the desired relative priority.

\section{PREMA MODIFICATIONS FOR HIDDEN TERMINALS}

In this section we extend the analysis of PREMA with simulations in an ad hoc environment with hidden and exposed

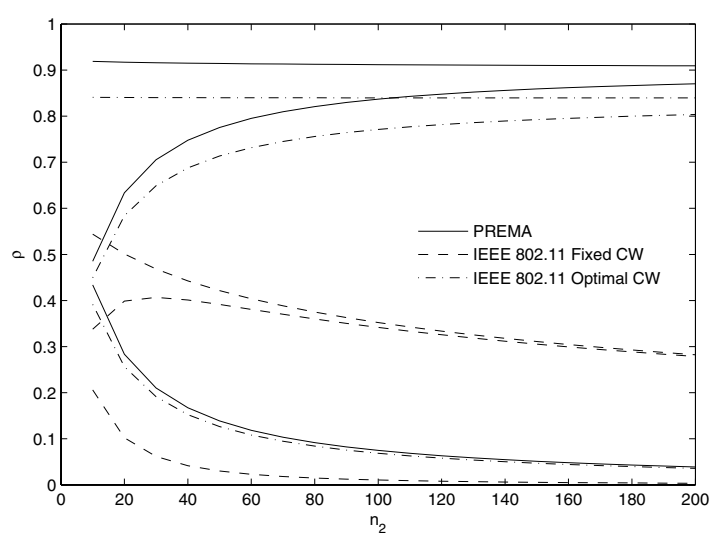

(a) $n_{1}=50, n_{2}=$ variable

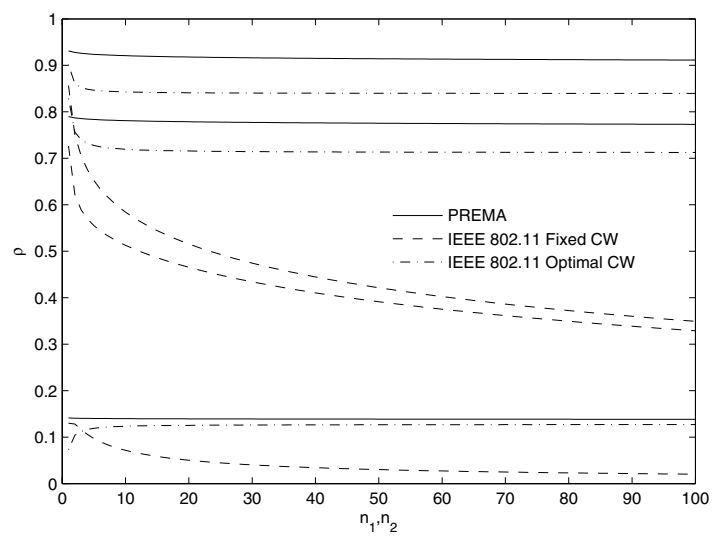

(b) $n_{1}=n_{2}=$ variable

Fig. 6. Overall channel utilization and channel utilization per class with two priority classes. From top to bottom for each protocol: Overall channel utilization, channel utilization of high priority traffic and channel utilization for low priority traffic.

terminals. Plain PREMA and two versions adapted for hidden terminals are compared to IEEE 802.11 with RTS/CTS.

Previously, we have compared PREMA to bursting, treesplitting and CSMA/CA protocols. Here, no comparison was made with DQRAP-DW since it does not work well with hidden terminals. First, DQRAP-DW requires $(0 / 1 / m)$ feedback which might not be correctly available with hidden terminals. Second, DQRAP-DW is based on a global, implicit, shared state and with hidden terminals a node can be part of two or more such shared states.

We propose two ways to modify PREMA to cope with hidden terminals. The first, PREMA- $\alpha$ : is to perform an RTS/CTS exchange after a (seemingly) successful contention. The second, PREMA- $\beta$, is to replace the elimination bursts with proper RTS packets.

In PREMA- $\beta$, each slot is divided into two parts. In the first part, each node randomly selects to transmit, or not, an RTS packet. Non-transmitting nodes sense the channel and leave the contention if it is busy. In the second part, nodes transmit CTS packets as appropriate. $(0 / 1 / m)$ feedback is obtained. Unless a node receives a CTS packet it remains in the contention for a maximum of $h$ eliminations. This method 
has similarities with CARMA/FS [13], which is a deterministic tree-splitting protocol where the winner is selected using depth-first traversal of a node ID tree.

\section{A. Scenario}

The effect of hidden terminals depends on the number of nodes within interference/transmission range of the receiver. If two nodes, not within detection range, start their transmissions concurrently to one common receiver the transmissions will overlap and most likely corrupt the data. A collision may only be detected from ARQ feedback at the end of the transmission and this makes collisions very costly in terms of capacity loss.

The carrier sense threshold determines the lower bound for which a signal causes the radio to indicate a busy channel. The threshold translates to a distance, the detection range, denoted by $R_{c s}$, depending on path loss and fading. The detection range has a large impact on the number of hidden terminals. If the threshold is sufficiently low, few nodes will be hidden from the sender and a collision is less likely. However, a low threshold will cause more nodes to be exposed to a transmission and thus prevent efficient reuse of the channel [25]. We have tested two different values for the detection range: 1 and 1.41 times the transmission range $\left(R_{t x}\right)$.

Here we consider a square simulation area with sides 1200 meters long and 50 nodes. Each sender transmits packets according to a Poisson process with rate $\lambda$ packets per second. The receiver for each transmission is uniformly chosen among the neighbors of the sender. With ad-hoc multi-hop routing receivers might be chosen differently.

\section{B. Simulation Results}

In the first simulation scenario all protocols are evaluated under saturation conditions with different $R_{t x}$ and $R_{c s}$. In the next scenario the aggregated load is gradually increased by increasing $\lambda$ for each individual sender until the saturation point is reached. In both scenarios the aggregated throughput is measured.

In Fig. 7, all protocols show lower aggregated throughput for higher values of the transmission power, showing that the spatial re-use of the channel is decreasing. The same happens when $R_{c s}$ increases.

PREMA- $\beta$ generally achieves the lowest throughput, mainly because of the high overhead in continuously transmitting RTS packets to resolve the conflicts, see Fig. 7(a). Performance is lowest when $R_{t x}=R_{c s}$. If multiple conflicts are ongoing, in different areas, the generated interference will be high and the probability of a successful RTS/CTS exchange very low. A higher $R_{c s} / R_{t x}$ ratio will increase the size of the conflict areas leading to fewer parallel contentions.

The rate of decline is increasing somewhat for all protocols around $11 \mathrm{dBm}$ as a result of the two-ray path loss model. For smaller values of $R_{t x}$ all nodes will experience free space propagation but at the Fresnel break point, nodes experience a stronger attenuation. This causes first order discontinuity in the relationship between transmission power and $R_{t x}$.
Comparing the performance of PREMA in Fig. 7(b) and 7(a) show that the use of the RTS/CTS handshake prior to the data transmission leads to an increased aggregated throughput, especially when $R_{c s}=1.41 R_{t x}$. IEEE 802.11 on the other hand shows no improvement or a slight decrease in throughput. For $R_{c s}=R_{t x}$ both protocols show an increase in throughput, but slightly larger for PREMA.

One conclusion from this scenario is that PREMA is more vulnerable to hidden terminals than IEEE 802.11. This result is not unexpected since PREMA is a "noisy" protocol and under high load conditions the channel will not be idle for very long periods due to frequent jamming transmissions. IEEE 802.11 on the other hand, has longer periods when the channel is idle as a result of the exponential back off.

PREMA achieves higher throughput than IEEE 802.11 for lower loads, while the opposite is true for higher loads, see Fig. 7(c). In this study, a transmission power of $12 \mathrm{dBm}$ is used. IEEE 802.11 and PREMA- $\beta$ show a similar small increase in throughput when the load is increased. This is a result of the capture effect.

PREMA does not show the same behavior for higher values of the load as the rest of the protocols. When $R_{c s}=R_{t x}$, the throughput is decreasing and for $R_{c s}=1.41 R_{t x}$ there is no or very little change in throughput. When increasing the load, the number of contenders is increasing causing more interference and lower throughput. This is clearly one drawback with the jamming transmissions used in PREMA.

\section{CONCLUSION}

We have proposed PREMA, a novel protocol for channel allocation based on repeated elimination bursts.

PREMA is most similar to EY-NPMA in that it uses bursts with random lengths according to the geometric distribution to eliminate nodes from channel access. The first difference is that the bursts are untruncated, allowing a very large number of nodes [8]. Using untruncated eliminations and a non-fixed bursting probability allows us to provide relative priorities whereas EY-NPMA only provides non-preemptive absolute priorities. The second difference is that instead of yielding, PREMA uses repeated eliminations allowing fine tuning of the success probability.

Analysis and simulations show that PREMA has very high channel utilization and success probability. The memoryless nature of PREMA - each contention is independent from the previous - results in good fairness properties compared to IEEE 802.11.

Relative and absolute prioritization is straightforward and does not significantly diminish channel utilization compared to using fixed contention windows in IEEE 802.11. Optimal contention windows in IEEE 802.11 show better performance than the standard mechanism but we argue that they are unrealistic because of the problems inherent in predicting future traffic.

Two extensions to PREMA, $\alpha$ and $\beta$, based on RTS/CTS for coping with hidden terminals were presented and simulated. The extended protocols are significantly better than the basic 


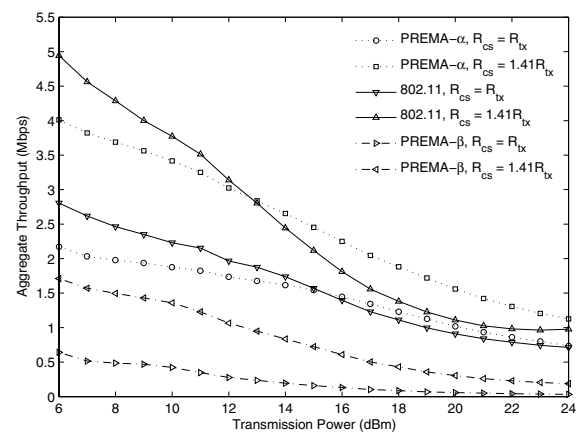

(a) RTS/CTS Access

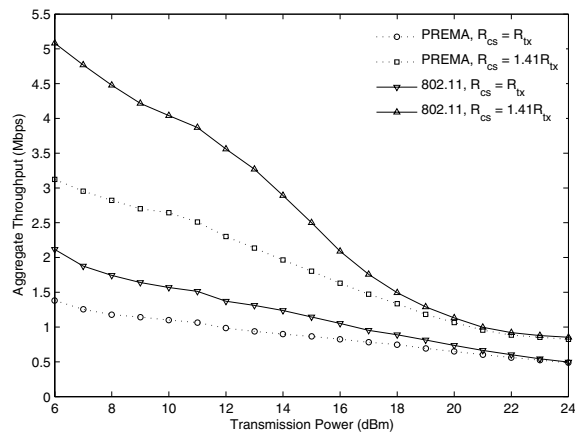

(b) Basic Access

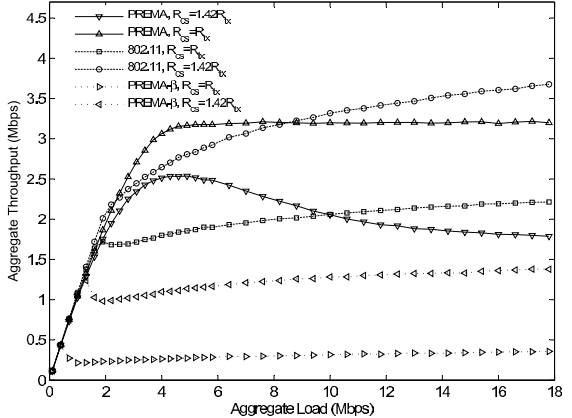

(c) Throughput as a function of aggregate load

Fig. 7. Aggregate throughput as a function of transmission power and aggregate load for different values of $R_{c s}$.

mechanism and IEEE 802.11 with RTS/CTS, especially under low load conditions. Under higher loads, IEEE 802.11 shows better performance than both PREMA- $\alpha$ and $-\beta$.

A further contribution of this paper is a version of DQRAP for decentralized wireless networks - DQRAP-DW. It performs very well in terms of fairness and has low but stable channel utilization.

In the future, we will work to improve performance under hidden terminal conditions. We will also look at higher layers and analyze TCP/IP traffic over PREMA. Further studies include comparison with the IEEE 802.11e standard. Multicast traffic is important, but has poor support from existing standards. We will look at ways to integrate multicast over PREMA with unicast over IEEE 802.11.

\section{REFERENCES}

[1] T. Nilsson, G. Wikstrand, and J. Eriksson, "A collision detection method for multicast transmissions in CSMA/CA networks," Wireless Communications and Mobile Computing, vol. 7, no. 6, pp. 795-808, 2006.

[2] B. O'Hara, Ed., Wireless LAN Medium Access Control (MAC) and Physical Layer (PHY) Specifications. New York, NY, USA: IEEE, 1999.

[3] C. Wang, B. Li, and L. Li, "A new collision resolution mechanism to enhance the performance of IEEE 802.11 DCF," IEEE Transactions on Vehicular Technology, vol. 53, no. 4, pp. 1235-1246, July 2004.

[4] Y. C. Tay and K. C. Chua, "A capacity analysis for the IEEE 802.11 MAC protocol," Wireless Networks, vol. 7, no. 2, pp. 159-71, 2001.

[5] F. Calì, M. Conti, and E. Gregori, "Dynamic tuning of the IEEE 802.11 protocol to achieve a theoretical throughput limit," IEEE/ACM Trans. Netw., vol. 8, no. 6, pp. 785-799, 2000.

[6] L. Yun, L. Ke-Ping, Z. Wei-Liang, and C. Qian-Bin, "A novel random backoff algorithm to enhance the performance of IEEE 802.11 DCF," Wireless Personal Communications, vol. 36, no. 1, pp. 29-44, 2006.

[7] Broadband Radio Access Networks (BRAN); HIgh PErformance Radio Local Area Network (HIPERLAN) Type 1; Functional Specification, EN 300652 V1.2.1 ed., ETSI, F-06921 Sophia Antipolis Cedex, France, July 1998 .

[8] G. Wikstrand and T. Nilsson, "Untruncated eliminations in the EYNPMA MAC protocol: Performance and optimality," IEEE Communications Letters, vol. 11, no. 2, pp. 213-215, Feb 2007.

[9] J. L. Sobrinho and A. S. Krishnakumar, "Real-time traffic over the IEEE 802.11 medium access control layer," Bell Labs Technical Journal, vol. 1, no. 2, pp. 172-87, 1996.

[10] J. I. Capetanakis, "Generalized TDMA: the multi-accessing tree protocol," IEEE Transactions on Information Theory, vol. COM-27, no. 10, pp. 1476-84, October 1979.
[11] W. Xu and G. Campbell, "A distributed queueing random access protocol for a broadcast channel," SIGCOMM Comput. Commun. Rev., vol. 23 , no. 4, pp. 270-278, 1993.

[12] H. J. Lin and G. Campbell, "Using DQRAP (distributed queueing random access protocol) for local wireless communications," in Proceedings of Wireless '93, 1993, pp. 625-635.

[13] R. Garcés and J. J. Garcia-Luna-Aceves, "Collision avoidance and resolution multiple access: First-success protocols," in IEEE Int. Conf. on Communications, vol. 2. IEEE, June 1997, pp. 599-703.

[14] W. Szpankowski and V. Rego, "Yet another application of a binomial recurrence: Order statistics," Computing, vol. 43, pp. 401-10, 1990.

[15] P. Kirschenhofer and H. Prodinger, "The number of winners in a discrete geometrically distributed sample," Annals of Applied Probability, vol. 6, no. 2, pp. 687-94, 1996.

[16] T. Janczak and J. Wozniak, "Modified EY-NPMA channel access scheme," Electronics Letters, 2001.

[17] A. J. E. M. Janssen and M. J. de Jong, "Analysis of contention tree algorithms," IEEE Transactions on Information Theory, vol. 46, no. 6 , pp. 2163-72, September 2000.

[18] G. Dimitriadis and F.-N. Pavlidou, "Two alternative schemes to EYNPMA for medium access in high bitrate wireless LANs," Wireless Personal Communications, vol. 28, pp. 121-142, 2004.

[19] G. Bianchi, "Performance analysis of the IEEE 802.11 distributed coordination function," IEEE Journal on Selected Areas in Communications, vol. 18, no. 3, pp. 535-47, March 2000.

[20] X. Zeng, R. Bagrodia, and M. Gerla, "Glomosim: A library for the parallel simulation of large-scale wireless networks," in PADS '98. Washington, DC, USA: IEEE Computer Society, 1998, pp. 154-61.

[21] R. K. Jain, D.-M. W. Chiu, and W. R. Hawe, "A quantitative measure of fairness and discrimination for resource allocation in shared computer systems," Digital Equipment Corporation, 77 Reed Road, Hudson, MA 01749, US, Tech. Rep. DEC-TR-301, September 1984.

[22] Y. Xiao, "A simple and effective priority scheme for IEEE 802.11," IEEE Communications Letters, vol. 7, no. 2, pp. 70-72, February 2003.

[23] Y. Ge, J. C. Hou, and W. Choi, "An analytic study of tuning systems parameters in IEEE 802.11e enhanced distributed channel access," Computer Networks, vol. 51, no. 8, pp. 1955-1980, June 2007.

[24] T. Cole, Ed., Wireless LAN Medium Access Control (MAC) and Physical Layer (PHY) Specifications, Amendment 8: Medium Access Control (MAC) Quality of Service Enhancements. New York, NY, USA: IEEE, 2005.

[25] J. Zhu, X. Guo, L. L. Yang, W. S. Conner, S. Roy, and M. M. Hazra, "Adapting physical carrier sensing to maximize spatial reuse in 802.11 mesh networks," Wireless Communications and Mobile Computing, vol. 4 , no. 8, pp. 933-946, 2004. 